\title{
Proposals for a Reappraisal of the Status of the Names of Plant-Pathogenic Pseudomonas Species
}

\author{
D. W. DYE, J. F. BRADBURY, R. S. DICKEY, M. GOTO, C. N. HALE, A. C. HAYWARD, \\ A. KELMAN, R. A. LELliOTT, P. N. PATEL, D. C. SANDS, M. N. SCHROTH, D. R. W. \\ WATSON, AND J. M. YOUNG
}

Plant Diseases Division, Auckland, New Zealand

\begin{abstract}
Two lists of phytopathogenic Pseudomonas species are presented. The first list comprises 80 names of species that have been validly published and for which cultures are available. It is proposed that these names be retained until a comprehensive rational classification is produced for this group. The second list contains more than 140 names which it is proposed should be rejected on grounds of illegitimacy, synonymy, lack of authentic cultures, or inappropriate classification.
\end{abstract}

A complete revision of the genus Pseudomonas Migula 1894 is presented in Bergey's Manual (27). In this revision there are only seven descriptions of plant-pathogenic species, namely, $P$. fluorescens Migula 1895 (some strains of biotype II), P. syringae van Hall 1902, $P$. cichorii (Swingle 1925) Stapp 1928, $P$. caryophylli (Burkholder 1942) Starr and Burkholder 1942, $P$. cepacia Burkholder 1950, $P$. marginata (McCulloch 1921) Stapp 1928, and P. solanacearum (Smith 1896) Smith 1914. While many plant bacteriologists would probably agree that on present evidence most plantpathogenic Pseudomonas nomenspecies do not justify ranking as taxospecies and that their names can, if nomenclaturally valid, be regarded as synonyms of one or another of the seven taxospecies recognized by Doudoroff and Palleroni, they would also consider that the treatment of this genus by Doudoroff and Palleroni (27) is inadequate from a plant pathologist's viewpoint because it does not provide a nomenclature that expresses the phytopathogenic individuality of these bacteria (30). Whatever the taxonomic and other disadvantages of the existing nomenclature of the plant-pathogenic pseudomonads, it reflects the pathogenic properties of these organisms better than the nomenclature used in Bergey's Manual. It therefore seems to us important to retain those names that are nomenclaturally legitimate until a satisfactory rationalization can be made that combines a "general purpose" classification, such as that of Doudoroff and Palleroni (27), with a "special purpose" classification catering to pathologists; the position of the species in the related genus Xanthomonas is similar $(31,50)$.

The International Code of Nomenclature of Bacteria approved by the International Com- mittee on Systematic Bacteriology at the First Congress of the Bacteriology Section of the International Association of Microbiological Societies in Jerusalem in 1973 (in press) provides that "names of bacteria in the various categories validly published up to 31 December 1977 will be assessed by the Judicial Commission with the assistance of taxonomic experts." "When approved by the ICSB these Approved Lists of Bacterial Names will be published in the IJSB before 1 January 1980 . Names validly published under the Code between 1 January 1978 and 1 January 1980 will be added to the Approved Lists" but "those names validly published prior to 1 January 1980 and not included in the lists will have no further standing in nomenclature. They will not be added to the lists of nomina rejicienda and will thus be available for reuse for the naming of new taxa." Essential features of the names to be recorded in the approved lists are that they be validly published and that they refer to organisms for which authentic cultures are available in recognized culture collections. Although more than 220 names have been used for plant-pathogenic bacteria in the genus Pseudomonas Migula 1894, some are illegitimate because they are contrary to the rules of the revised Code, whereas others refer to species for which no representative cultures are known to exist. Many even lack adequate original descriptions by which cultures could be identified reliably if they are isolated again.

As a first step towards a more rational and useful classification of the plant-pathogenic pseudomonads, lists are presented here. They divide the existing nomenspecies into: (1) those that are legitimate according to the approved rules of the Bacteriological Code and which we propose should be retained in general use until a 
nomenclature emerges with the degree of differentiation that is sought by plant bacteriologists and pathologists, and (2) those that should not be retained because they are nomenclaturally illegitimate, are later objective or generally accepted subjective synonyms of those in list 1 , are without extant cultures in recognized collections, or have been inappropriately classified.

List 1. Pseudomonas nomenspecies proposed to be retained pending further research. It is acknowledged that some have received little attention and may well have been inappropriately classified. Therefore this list is likely to be modified before formal submissions are made for the Approved Lists of Bacterial Names. (Complete references are given for the authorities of these nomenspecies.)

P. aceris (Ark 1939) Starr and Burkholder 1942

$P$. agarici Young 1970

P. alboprecipitans Rosen 1922

$P$. alliicola (Burkholder 1942) Starr and Burkholder 1942

$P$. amygdali Psallidas and Panagopoulos 1974

$P$. andropogonis (Smith 1911) Stapp 1928

$P$. angulata (Fromme and Murray 1919) Stevens 1925

P. antirrhini Takimoto 1920

$P$. apii Jagger 1921

P. aptata (Brown and Jamieson 1913) Stevens 1925

P. asplenii (Ark and Tompkins 1946) Săvulescu 1947

P. atrofaciens (McCulloch 1920) Stevens 1925

P. azadirachtae Desai et al. 1966

P. barkeri (Berridge 1924) Dowson 1943

$P$. berberidis (Thornberry and Anderson 1931) Stapp 1935

$P$. betle (Ragunathan 1928) Săvulescu 1947

P. cannabina Sutić and Dowson 1959

$P$. caricapapayae Robbs 1956

$P$. caryophylli (Burkholder 1942) Starr and

Burkholder 1942

P. cattleyae (Pavarino 1911) Săvulescu 1947

$P$. cepacia Burkholder 1950

P. ciccaronei Ercolani and Caldarola 1972

$P$. cichorii (Swingle 1925) Stapp 1928

$P$. coronafaciens (Elliott 1920) Stevens 1925

$P$. coronafaciens subsp. atropurpurea

(Reddy and Godkin 1923) Stapp 1928

P. delphinii (Smith 1904) Stapp 1928

$P$. dysoxyli Hutchinson 1949

P. eriobotryae (Takimoto 1931) Dowson 1943

$P$. flectens Johnson 1956

P. floridana (Bourne 1970) Bourne 1970

$P$. garcae Amaral et al. 1956

$P$. glumae Kurita and Tabei 1967
P. glycinea Coerper 1919

$P$. helianthi (Kawamura 1934) Săvulescu 1947

P. hibiscicola Moniz 1963

P. lachrymans (Smith and Bryan 1915) Carsner 1918

P. lapsa (Ark 1940) Starr and Burkholder 1942

P. maculicola (McCulloch 1911) Stevens 1913

P. mangiferaeindicae Patel et al. 1948

P. marginalis (Brown 1918) Stevens 1925

P. marginata (McCulloch 1921) Stapp 1928

$P$. mellea Johnson 1923

P. melophthora Allen and Riker 1932

P. mori (Boyer and Lambert 1893) Stevens 1913

P. morsprunorum Wormald 1931

$P$. morsprunorum subsp. persicae Prunier et al. 1970

$P$. oryzicola Klement 1955

P. panici (Elliott 1923) Stapp 1928

$P$. papaveris Lelliott and Wallace 1955

P. papulans Rose 1917

P. passiflorae (Reid 1939) Burkholder 1948

$P$. pastinacae Burkholder 1960

P. phaseolicola (Burkholder 1926) Dowson 1943

P. pisi Sackett 1916

P. pomi Cole 1959

P. primulae (Ark and Gardner 1936) Starr and Burkholder 1942

P. ribicola Bohn and Maloit 1946

P. rubrilineans (Lee et al. 1925) Stapp 1928

$P$. rubrisubalbicans (Christopher and Edgerton 1930) Krasil'nikov 1949

P. savastanoi (Smith 1908) Stevens 1913

P. sesami Malkoff 1906

P. setariae (Okabe 1934) Săvulescu 1947

P. solanacearum (Smith 1896) Smith 1914

P. sorghicola Rangaswami et al. 1961

P. striafaciens (Elliott 1927) Starr and Burkholder 1942

$P$. syringae van Hall 1902

P. syringae subsp. capsici (Orsini 1942) Klement 1956

P. tabaci (Wolf and Foster 1917) Stevens 1925

$P$. tagetis Hellmers 1955

P. theae (Hori 1915) Okabe and Goto 1955

P. tolaasii Paine 1919

P. tomato (Okabe 1933) Alstatt 1944

P. ulmi Šutić and Tešić 1958

$P$. viburni (Thornberry and Anderson 1931)

Stapp 1935

P. viridiflava (Burkholder 1930) Dowson 1939

P. viridilivida (Brown 1915) Stevens 1925

P. viticola Nayudu 1972

P. vitiswoodrowii Patel and Kulkarni 1951

P. washingtoniae (Pine 1943) Elliott 1951

P. woodsii (Smith 1911) Stevens 1925 
List 2. Names of Pseudomonas species that it is proposed should not be retained because they are: (a) illegitimate; (b) objective synonyms or generally accepted subjective synonyms of names in list 1 ; (c) not represented by authentic cultures in recognized culture collections (most are also so inadequately described as to be unrecognizable again, i.e., they are hyponyms); (d) considered on the basis of cultures and/or descriptions not to be appropriately classified in the genus. (The literature citations are not given for authors of these nomenspecies.)

P. acernea Ogawa 1937 (c)

$P$. aleuritidis (McCulloch and Demaree 1932) Stapp 1935 (c)

P. allii (Griffiths 1887) Migula 1900 (c) (c)

P. aloes (Passalacqua 1929) Krasil'nikov 1949

P. amaranti Smith 1901 (c)

$P$. ananas Serrano 1934 (c)

$P$. araliae Uyeda 1909 (c)

P. astragali (Takimoto 1930) Săvulescu 1947 (c)

P. aucubicola Trapp 1936 (c)

$P$. aurantiaca tingitana (Remlinger and

Bailly 1935) Krasil'nikov 1949 (c)

$P$. avenae Manns 1909 (c)

P. batatae Cheng et al. 1962 (c)

$P$. betae (Wieringa 1927) Dowson 1943 (c)

$P$. bowlesiae (Lewis and Watson 1927) Dowson 1943 (c)

$P$. brassicae acidae (Lehman and Conrad 1896) Gruber 1909 (c)

P. brassicaevora (Delacroix 1905) Krasil'nikov 1949 (c) (c)

P. briosii (Pavarino 1915) Krasil'nikov 1949

P. burgeri (Potebnia 1915) Korobko and Nikiforuk 1972 (c)

P. bussei (Migula 1900) Krasil'nikov 1949 (c)

P. calendulae (Takimoto 1936) Dowson 1943 (c)

P. cannabina var. italica Goidanich and Ferri 1960 (a)

$P$. caryocyanea Dupaix 1933 (a)

$P$. castaneae (Kawamura 1934) Săvulescu

1947 (c)

$P$. caucasicum Shneider 1954 (c)

$P$. caulivorae (sic) (Prillieux and Delacroix 1890) Krasil'nikov 1949 (c)

P. cerasi Griffin 1911 (b)

P. cerasi var. prunicola Wilson 1933 (b)

$P$. cerasi var. pyri D'yakova 1961 (b)

$P$. derasi uraggi Sackett 1925 (a)

$P$. cerealia (Gentner 1920) Stapp 1928 (c)

P. cinnamona Miyake and Tsunodo 1938 (c) (c)

P. cissicola (Takimoto 1939) Burkholder 1948

P. citrarefaciens (Lee 1917) Stapp 1928 (b)

P. citriputealis (Smith 1913) Stapp 1928 (b)

$P$. coli-capsici (Passalacqua 1932) Krasil'nikov 1949 (c)

P. colurnae (Thornberry and Anderson 1937)

Burkholder 1948 (c)

$P$. conjac Uyeda 1910 (d)

P. corylii (Brzezinski 1903) Krasil'nikov 1949 (c)

P. cuboniani (Macchiati 1892) Krasil'nikov 1949 (c)

P. cumini (Kovachevsky 1936) Dowson 1943 (c)

P. cyamopsicola Rangaswami and Gowda 1963 (d)

$P$. desaiana (Burkholder 1939) Săvulescu

1947 (c)

P. destructans Potter 1899 (c)

$P$. dianthi (Arthur and Bolley 1896) Smith 1901 (c)

P. eleusineae Billimoria and Hegde 1971 (c)

$P$. endiviae Kotte 1930 (b)

$P$. erodii Lewis 1914 (c)

$P$. exitiosa Gardner and Kendrick 1921 (c)

P. fabae (Yu 1936) Burkholder 1948 (c)

P. fici (Cavara 1905) Krasil'nikov 1949 (d)

$P$. fluorescens var. antirrhinastrini Moffett 1966 (b)

$P$. fluorescens var. exitiosus van Hall 1903 (c)

$P$. formosanum (Okabe 1935) Krasil'nikov 1949 (c)

P. fragariae I Gruber 1902 (a)

P. fraxini (Brown 1932) Škorić 1938 (c)

$P$. gardeniae (Burkholder and Pirone 1941)

Dowson 1943 (c)

$P$. gardneri Sutić 1957 (d)

P. gardneri var. capsici Sutić 1957 (c)

$P$. gladioli Severini 1913 (c)

P. glycinea var. japonica (Takimoto 1927)

Săvulescu 1947 (b)

$P$. helianthi var. tuberosi Thornberry and

Anderson 1937 (b)

$P$. hemmianus (Yamamoto 1951) Okabe and Goto 1955 (c)

P. hibisci (Nakada and Takimoto 1923) Stapp 1928 (b)

(a)

P. hibiscicola Rangaswami and Gowda 1963

P. holci Kendrick 1926 (c)

$P$. hordei (Goto and Nakanishi 1951) Okabe and Goto 1955 (c)

$P$. hypertrophicans Stahel 1933 (d)

P. intybi (Swingle 1925) Stapp 1928 (b)

P. iridicola (Takimoto 1931) Stapp 1935 (c)

P. iridis van Hall 1902 (c) 
P. itoana Tochinai 1932 (d)

$P$. jaggeri Stapp 1928 (b)

P. koroiensis (sic) Uyeda 1908 (c) (c)

P. lacerans (Migula 1900) Krasil'nikov 1949

(c)

P. lachrymans f. sp. cucumis Gorlenco 1961

P. lachrymans f. sp. melonis Yatsynina 1939 (c)

P. lauracearum Harvey 1952 (c)

$P$. leguminiperdus (sic) (von Oven 1906) Stevens 1913 (c)

P. levistici Osterwalder 1909 (c)

P. lignicola Westerdijk and Buisman 1929 (c) (c)

$P$. ligustri (d'Oliveira 1936) Săvulescu 1947

P. lohnisii (sic) (Kalantarian 1925) Krasil'nikov 1949 (c)

P. lupini Bel'tyukova and Korol'ova 1968 (c)

P. lycopersici (Burgwitz 1924) Stapp 1928 (c)

P. lycopersicum (Groenewege 1912) Krasil'nikov $1949(c)$ (c)

P. madrasensis Rangaswami and Gowda 1963

P. maidis (Maiocchi 1881) Migula 1900 (c)

P. martyniae (Elliott 1925) Stapp 1928 (c)

$P$. matthiolae (Briosi and Pavarino 1912)

Dowson 1943 (c)

P. maublancii (Foex and Lansade 1936) Săvulescu 1947 (c)

$P$. medicaginis Sackett 1910 (c)

$P$. medicaginis var. phaseolicola (Burkholder 1926) Stapp and Kotte 1929 (b)

P. mori var. huszi Klement et al. 1960 (b)

P. musae Gäumann 1921 (c)

$P$. necrosis (Kalinenko 1933) Krasil'nikov 1949 (c)

$P$. nectarophila (Doidge 1917) Burkholder 1939 (c)

P. pachyrrhizus Fang and Ren 1960 (c)

$P$. panacis (Nakata and Takimoto 1922) Dowson 1943 (c)

P. panicimiliacei (Ikata and Yamauchi 1931) Săvulescu 1947 (c)

$P$. papaveris (Takimoto 1935) Okabe and Goto 1955 (c)

$P$. petasitis (Takimoto 1927) Săvulescu 1947 (c)

P. phormicola (Takimoto 1933) Krasil'nikov 1949 (c)

P. pini (Chester 1897) Petri 1924 (c)

P. polycolor Clara 1930 (b)

P. polygoni (Thornberry and Anderson 1937)

Burkholder 1948 (c)

P. prunicola Wormald 1930 (b) (c) (b)

P. punctulans (Bryan 1933) Săvulescu 1947

$P$. putrefaciens liquefaciens (Delacroix 1906)

Krasil'nikov 1949 (a)

P. putrefaciens putridus (Delacroix 1906)

Krasil'nikov 1949 (a)

P. pyri (D'yakova 1955) Gorlenco 1961 (c)

$P$. radiciperda (Zhavoronkova 1932) Săvulescu 1947 (c)

P. rhizoctonia (Thomas 1922) Stevens 1925 (c)

$P$. ricini (Archibald 1927) Robbs 1954 (b)

$P$. rimaefaciens Koning 1938 (b)

P. rubi idaei Natal'ina et al. 1954 (d)

$P$. saliciperda Lindeijer 1932 (c)

P. sambuci Tešić and Todorović 1953 (c)

P. savastanoi var. fraxini (Brown 1932) Dowson 1943 (b)

P. savastanoi var. nerii Smith 1928 (b)

P. seminum Cayley 1917 (d)

P. siccata (Moniz and Patel 1958) Rangaswami 1962 (c)

P. sojae (Wolf 1920) Stapp 1928 (c)

P. sojae var. japonica (Takimoto 1927) Krasil'nikov 1949 (b)

$P$. solanacearum var. asiatica (Smith 1914) Stapp 1928 (b)

P. solaniolens Paine 1923 (c)

P. sorghi (Burrill 1887) Krasil'nikov 1949 (c)

P. spongiosa (Aderhold and Ruhland 1905)

Braun 1927 (b)

P. stizolobii (Wolf 1920) Stapp 1935 (b)

$P$. straminea Komagata 1961 (c)

P. striafaciens var. japonica Mukoo 1955 (c)

P. suberfaciens (Burr 1928) Krasil'nikov 1949 (c)

P. syringae var. papulans (Rose 1917) Smith 1944 (c)

$P$. syringae f. sp. populea Sabet and Dowson 1952 (b)

P. tectonae Roldan and Andres 1954 (c)

P. tonelliana (Ferraris 1926) Burkholder 1948 (b)

P. trifolii Huss 1907 (d)

P. trifoliorum (Jones et al. 1923) Stapp 1928 (b)

P. utiformica Clara 1932 (b)

$P$. viciae Uyeda 1915 (c)

$P$. vignae Gardner and Kendrick 1923 (b)

$P$. vignae var. leguminophila (Burkholder 1930) Magrou and Prévot 1948 (c)

$P$. viridifaciens (Tisdale and Williamson 1923) Tisdale and Williamson 1923 (b)

$P$. viridiflava var. concentrica (Petersen 1932) Săvulescu 1947 (c)

P. wieringae (Elliott 1930) Săvulescu 1947 (c)

P. xanthochlora (Schuster 1913) Stapp 1928

(c) 


\section{P. zingiberi Uyeda 1909 (c)}

\section{REPRINT REQUESTS}

Address reprint requests to: Dr. Douglas W. Dye, Plant Diseases Division, Private Bag, Auckland, New Zealand.

\section{LITERATURE CITED}

1. Allen, T. C., and A. J. Riker. 1932. A rot of apple fruit caused by Phytomonas melophthora n.sp. following invasion by the apple maggot. Phytopathology 22:557-571.

2. Alstatt, G. E. 1944. Tomato diseases in Texas. Plant Dis. Rep. 28:530.

3. Amaral, J. F. do, C. Teixeira, and E. D. Pinheiro. 1956. $\mathrm{O}$ bactério causador da mancha aureolada do Cafeeiro. Arq. Inst. Biol. Sao Paulo 23:151-155.

4. Ark, P. A. 1939. Bacterial leaf spot of maple. Phytopathology 29:968-970.

5. Ark, P. A. 1940. Bacterial stalk rot of field corn caused by Phytomonas lapsa n.sp. Phytopathology 30:1.

6. Ark, P. A., and M. W. Gardner. 1936. Bacterial leaf spot of Primula. Phytopathology 26:1050-1055.

7. Ark, P. A., and C. M. Tompkins. 1946. Bacterial leaf blight of birds-nest fern. Phytopathology 36:758-761.

8. Berridge, E. M. 1924. The influence of hydrogen ion concentration on the growth of certain bacterial plant parasites and saprophytes. Ann. Appl. Biol. 11:73-85.

9. Bohn, G. W. and J. C. Maloit. 1946. Bacterial spot of native golden currant (Ribes aureum). J. Agric. Res. Washington, D.C. 73:281-290.

10. Bourne, B. A. 1970. Studies on the bacterial red stripe disease of sugarcane in Florida. Sugarcane Pathol. Newslett. 4:27-33.

11. Bourne, B. A. 1970. Supplemental notes on the causal bacterium of sugarcane red stripe disease in Florida. Sugarcane Pathol. Newslett. 5:40-42.

12. Boyer, G., and F. Lambert. 1893. Sur deux nouvelles maladies du mùrier. C. R. Acad. Sci. 117:342-343

13. Brown, N. A. 1915. A bacterial disease of lettuce. J. Agric. Res. Washington, D. C. 4:475-478.

14. Brown, N. A. 1918. Some bacterial diseases of lettuce. J. Agric. Res. Washington, D. C. 13:367-388.

15. Brown, N. A., and C. O. Jamieson. 1913. A bacterium causing a disease of sugar-beet and nasturtium leaves. J. Agric. Res. Washington, D. C. 1:189-210.

16. Burkholder, W. H. 1926. A new bacterial disease of the bean. Phytopathology 16:915-928.

17. Burkholder, W. H. 1930. The bacterial diseases of bean: a comparative study. Mem. Cornell Univ. Agric. Exp. Sta. 127:1-88.

18. Burkholder, W. H. 1942. Three bacterial plant pathogens. Phytomonas caryophylli sp.n, Phytomonas al. liicola sp.n and Phytomonas manihotis (Arthaud-Berthet et Bondar) Viegas. Phytopathology 32:141-149.

19. Burkholder, W. H. 1948. Genus I. Pseudomonas Migula, p. 138. In R. S. Breed, E. G. D. Murray, and A. P. Hitchens (ed.), Bergey's manual of determinative bacteriology, 6th ed. Baillière, Tindall \& Cox, London.

20. Burkholder, W, H. 1950. Sour skin, a bacterial rot of onion bulbs. Phytopathology 40:115-117.

21. Burkholder, W. H. 1960. A bacterial brown rot of parsnip roots. Phytopathology 50:280-282.

22. Carsner, E. 1918. Angular-leafspot of cucumber: dissemination, over-wintering and control. J. Agric. Res. Washington, D.C. 15:201-220.

23. Christopher, W. N., and C. W. Edgerton. 1930. Bacterial stripe diseases of sugar-cane in Louisiana. J. Agric. Res. Washington, D.C. 41:259-267.

24. Coerper, F. M. 1919. Bacterial blight of soybean. J. Agric. Res. Washington, D.C. 18:179-194.

25. Cole, M. 1959. Bacterial rotting of apple fruit. Ann.
Appl. Biol. 47:601-611.

26. Desai, S. G.. A. B. Gandhi, M. K. Patel, and W. V Kotasthanae. 1966. A new bacterial leaf spot and blight of Azadirachta indica A. Juss. Indian Phytopathol. 19:322-323.

27. Doudoroff, M., and N. J. Palleroni. 1974. Genus I. Pseudomonas Migula 1894, 237 Nom. cons. Opin. 5, Jud. Comm. 1952, 121, p. 217-243. In R. E. Buchanan and N. E. Gibbons (ed.), Bergey's manual of determinative bacteriology, 8 th ed. The Williams \& Wilkins Co., Baltimore.

28. Dowson, W. J. 1939. On the systematic position and generic names of the Gram negative bacterial plant pathogens. Zentralbl. Bakteriol. Parasitenkd. Infektionskr. Hyg. Abt. 2 100:177-193.

29. Dowson, W. J. 1943. On the generic names Pseudomo nas, Xanthomonas and Bacterium for certain bacterial plant pathogens. Trans. Br. Mycol. Soc. 26:4-14.

30. Dye, D. W., et al. 1974. The problem of nomenclature of the plant pathogenic pseudomonads. Rev. Plant $\mathrm{Pa}$ thol.. 53:953-962.

31. Dye, D. W., and R. A. Lelliott. 1974. Genus II. Xanthomonas Dowson 1939, 187, p. 243-249. In R. E. Buchanan and N. E. Gibbons (ed.), Bergey's manual of determinative bacteriology, 8th ed. The Williams \& Wilkins Co., Baltimore.

32. Elliott, C. 1920. Halo blight of oats. J. Agric. Res. Washington, D.C. 19:139-172.

33. Elliott, C. 1923. A bacterial stripe disease of proso millet. J. Agric. Res. Washington, D.C. 26:151-160.

34. Elliott, C. 1927. Bacterial stripe blight of oats. J. Agric. Res. Washington, D.C. 35:811-824.

35. Elliott, C. 1951. Manual of bacterial plant pathogens, 2nd ed. Chronica Botanica Co., Waltham, Mass.

36. Ercolani, G. L., and M. Caldarola. 1972. Pseudomonas Ciccaronei sp.n. agente di una maculatura fogliare del carrubo in Puglia. Phytopathol. Mediterr. $11: 71-73$.

37. Fromme, F. D., and T. J. Murray. 1919. Angular leafspot of tobacco, an underscribed bacterial disease. J. Agric. Res. Washington, D.C. 16:219-228.

38. Hellmers, E. 1955. Bacterial leaf spot of African Marigold (Tagetis erecta) caused by Pseudomonas tagetis sp.n. Acta Agric. Scand. 5:185-200.

39. Hori, S. 1915: An important disease of tea plants caused by a bacterium. J. Plant Protect. (Tokyo) 2:1-7.

40. Hutchinson, P. B. 1949. A bacterial disease of Dysoxylum spectabile caused by the pathogen Pseudomonas dysoxyli n.sp. N. Z. J. Sci. Technol. Sect. B 30:274-286.

41. Jagger, I. C. 1921. Bacterial leafspot disease of celery. J. Agric. Res. Washington, D.C. 21:185-188.

42. Johnson, J. 1923. A bacterial leafspot of tobacco. J. Agric. Res. Washington, D.C. 23:481-493.

43. Johnson, J. C. 1956. Pod twist: a previously unrecorded bacterial disease of French bean (Phaseolus vulgaris L.) Queensl. J. Agric. Sci. 13:127-158.

44. Kawamura, E. 1934. Bacterial leaf spot of sunflower. Ann. Phytopathol. Soc. Jpn. 4:25-28.

45. Klement, Z. 1955. A new bacterial disease of rice caused by Pseudomonas oryzicola n.sp. Acta Microbiol. Acad. Sci. Hung. 2:265-274.

46. Klement, Z. 1956. Bacterial soft rot in green pepper (Capsicum annuum). Acta Microbiol. Acad. Sci. Hung. 3:409-416.

47. Krasil'nikov, N. A. 1949. "Opredelitel' bakterii i ak tinomitsetov" (Key to bacteria and actinomycetes) Moskva-Leningrad. Izdatel'stvo Akademii Nauk SSSR, Moscow.

48. Kurita, T., and H. Tabei. 1967. On the pathogenic bacterium of bacterial grain rot of rice. Ann. Phytopathol. Soc. Jpn. 33:111.

49. Lee, H. A., H. A. Purdy, C. C. Barnum, and J. P. Martin. 1925. A comparison of red-stripe disease with 
bacterial diseases of sugar-cane and other grasses, $p$. 64-74. In Red-stripe disease studies. Experiment Station Hawaiian Sugar Planters Association, Honolulu.

50. Lelliott, R. A. 1972. The genus Xanthomonas, p. 269-272. In H. P. Maas Geesteranus (ed.), Centre for Agricultural Publishing and Documentation, Wageningen. Proc. 3rd Int. Conf. Plant Pathogenic Bacteria, Wageningen 1971.

51. Lelliott, R. A., and M. A. Wallace. 1955. A bacterial disease of Shirley poppies in Tanganyika. Trans. Br. Mycol. Soc. 38:88-91.

52. McCulloch, L. 1911. A spot disease of cauliflower. Bull. U. S. Bur. Plant Ind. 225:1-15.

53. McCulloch, L. 1920. Basal glume rot of wheat. J. Agric. Res. Washington, D.C. 18:543-551.

54. McCulloch, L. 1921. A bacterial disease of gladiolus. Science 54:115-116.

55. Malkoff, K. 1906. Weitere Untersuchungen über die Bakterienkrankheit auf Sesamum orientale. Zentralb. Bakteriol. Parasitenkd. Infektionskr. Abt. 2 16:664666.

56. Migula, W. 1894. Ueber ein neues System der Bakterien. Arb. Bakteriol. Inst. Tech. Hochsch. Karlsruhe $1: 235-238$.

57. Moniz, L. 1963. A leaf-spot of apple blossom. Curr. Sci. 32:177.

58. Nayudu, M. V. 1972. Pseudomonas viticola sp.nov. incitant of a new bacterial disease of grape vine. Phytopathol. Z. 73:183-186.

59. Okabe, N. 1933. Bacterial diseases of plants occurring in Formosa. IV. Bacterial brown-stripe of Italian millet. J. Soc. Trop. Agric. (Taiwan) 6:54-63.

60. Okabe, N. 1934. Bacterial diseases of plants occurring in Formosa. IV. J. Soc. Trop. Agric. (Taiwan) 6:54-63.

61. Okabe, N., and M. Goto. 1955. Bacterial plant diseases in Japan. IV. Studies on bacterial shoot blight of tea-plant caused by Pseudomonas theae n.sp. Rep. Fac. Agric. Shizuoka Univ. 5:96-99.

62. Orsini, G. 1942. A bacterial soft rot of Capsicum fruits. Int. Bull. Plant Protect. 33:33-36.

63. Paine, S. G. 1919. Studies in bacteriosis. II. A brown blotch disease of cultivated mushrooms. Ann. Appl. Biol. 5:206-219.

64. Patel, M. K., and Y. S. Kulkarni. 1951. A new bacterial leaf spot on Vitis woodrowii Stapf. Curr. Sci. 20:132.

65. Patel, M. K., L. Moniz, and Y. S. Kulkarni. 1948. A new bacterial disease of Mangifera indica L. Curr. Sci. 17:189-190.

66. Pavarino, G. L. 1911. Malattie causate da bacteri nelle orchidee. Atti R. Accad. Lincei 20:233-237.

67. Pine, L. 1943. A hitherto unreported disease of the Washington palm. Phytopathology 33:1201-1204.

68. Prunier, J. P., J. Luisetti, and L. Gardan. 1970. Etudes sur les bactérioses des arbres fruitiers. II. Caractérisation d'un Pseudomonas non-fluorescent agent d'une bactériose nouvelle du pêcher. Ann. Phytopathol. 2:181-197.

69. Psallidas, P. G., and C. G. Panagopoulos. 1974. A new bacteriosis of almond caused by Pseudomonas amyg. dali sp.nov. Ann. Inst. Phytopathol. Benaki, in press.

70. Ragunathan, C. 1928. Bacterial leaf spot of betel. Ann. R. Bot. Gard. Peradeniya 11:51-62.

71. Rangaswami, G., N. N. Prasad, and K. S. S. Easwaran. 1961. Two new bacterial diseases of sorghum. Andhra Agric. J. 8:269-272.

72. Reddy, C. S., and J. Godkin. 1923. A bacterial disease of brome-grass. Phytopathology 13:75-86.

73. Reid, W. D. 1939. Grease-spot of passion-fruit. N. Z. J. Sci. Technol. Sect. A 20:260-265.

74. Robbs, C. F. 1956. Uma nova doenca bacteriana do mamoeiro (Carica papaya L.) Rev. Soc. Bras. Agron.
12:73-76

75. Rose, D. H. 1917. Blister spot of apples and its relation to a disease of apple bark. Phytopathology 7:198-208.

76. Rosen, H. R. 1922. A bacterial disease of foxtail (Chaeto chloa lutescens). Ann. Mo. Bot. Gard. 9:333-402.

77. Sackett, W. G. 1916. A bacterial stem blight of field and garden peas. Bull. Colo. State. Univ. Agric. Exp. Stat. 218:1-43.

78. Săvulescu, T. 1947. Contribution à la classification des Bactériacées phytopathogènes. An. Acad. Române. Ser. III Tom 22 Mem. 4:1-26.

79. Smith, E. F. 1896. A bacterial disease of the tomato, eggplant and Irish potato (Bacillus solanacearum n.sp.). U.S. Dept. of Agriculture Bull. 12. U.S. Dept. of Agriculture, Washington, D.C.

80. Smith, E. F. 1904. Bacterial leaf spot diseases. Science Ser. 3 19:417-418.

81. Smith, E. F. 1908. Recent studies of the olive-tubercle organism, p. 25-43. U.S. Dept. of Agriculture Bull. 131. U.S. Department of Agriculture, Washington, D.C.

82. Smith E. F. 1911. Bacteria in relation to plant disease, vol. 2. Carnegie Institute, Washington, D.C.

83. Smith, E. F. 1914. Bacteria in relation to plant diseases, vol. 3. Carnegie Institute, Washington, D.C

84. Smith, E. F., and M. K. Bryan. 1915. Angular leaf-spot of cucumbers. J. Agric. Res. Washington, D.C. $5: 465-476$.

85. Stapp, C. 1928. Schizomycetes (Spaltpilze oder Bakterien), p. 1-295. In P. Sorauer (ed.), Handbuch der Pflanzenkrankheiten, vol. 2. Paul Parey, Berlin.

86. Stapp, C. 1935. Contemporary understanding of bacterial plant-diseases and their causal organisms. Bot. Rev. 1:405-425.

87. Starr, M. P., and W. H. Burkholder. 1942. Lipolytic activity of phytopathogenic bacteria determined by means of spirit blue agar, and its taxonomic significance. Phytopathology 32:598-604.

88. Stevens, F. L. 1913. The fungi which cause plant disease. The MacMillan Co., New York.

89. Stevens, F. L. 1925. Plant disease fungi. The MacMillan Co., New York.

90. Šutić, D., and W. J. Dowson. 1959. An investigation of a serious disease of Hemp (Cannabis sativa L.) in Jugoslavia. Phytopathol. Z. 34:307-314.

91. Sutić, D., and Z. Tešić. 1958. Jedna nova bakterioza Bresta izazivać Pseudomonas ulmi n.sp. Zast. Bilja 45:13-25.

92. Swingle, D. B. 1925. Center rot of "French endive" or wilt of chicory (Cichorium intybus L.). Phytopathology 15:730.

93. Takimoto, S. 1920. On the bacterial leaf-spot of Antirrhinum majus L. Bot. Mag. 34:253-257.

94. Takimoto, S. 1931. Bacterial bud rot of loquat. J. Plant Protect. (Tokyo) 18:349-355.

95. Thornberry, H. H., and H. W. Anderson. 1931. A bacterial disease of barberry caused by Phytomonas berberidis n.sp. J. Agric. Res. Washington, D.C. 43:29-36.

96. Thornberry, H. H., and H. W. Anderson. 1931. Bacterial leaf spot of Viburnum. Phytopathology 21:907-912.

97. van Hall, C. J. J. 1902. Bijdragen tot de kennis der Bakterieele Plantenziekten. Inaugural Dissertation. Coöperatieve Drukkerij-Vereeniging "Plantijn", Amsterdam.

98. Wolf, F. A., and A. C. Foster. 1917. Bacterial leaf spot of tobacco. Science Ser. 46:361-362.

99. Wormald, H. 1931. Bacterial diseases of stone fruit trees in Britain. III. The symptoms of bacterial canker in plum trees. J. Pomol. Hortic. Sci. 9:239-256.

100. Young, J. M. 1970. Drippy gill: a bacterial disease of cultivated mushrooms caused by Pseudomonas agarici n.sp. N. Z. J. Agric. Res. 13:977-990. 